\title{
Thin Filament-Reconstituted Skinned Muscle Fibers for the Study of Muscle Physiology
}

\author{
Sayaka Higuchi, ${ }^{1}$ Yoshikazu Tsukasaki, ${ }^{1}$ Norio Fukuda, ${ }^{2}$ \\ Satoshi Kurihara, ${ }^{2}$ and Hideaki Fujita ${ }^{1}$ \\ ${ }^{1}$ Laboratory for Comprehensive Bioimaging, Riken Quantitative Biology Center, OLABB, 6-2-3 Furuedai, Suita, \\ Osaka 565-0874, Japan \\ ${ }^{2}$ Department of Cell Physiology, The Jikei University School of Medicine, Tokyo 105-8461, Japan
}

Correspondence should be addressed to Hideaki Fujita, hideaki.fujita@riken.jp

Received 21 July 2011; Accepted 12 August 2011

Academic Editor: Henk Granzier

Copyright (c) 2011 Sayaka Higuchi et al. This is an open access article distributed under the Creative Commons Attribution License, which permits unrestricted use, distribution, and reproduction in any medium, provided the original work is properly cited.

\begin{abstract}
We review the use of thin filament-reconstituted muscle fibers in the study of muscle physiology. Thin filament extraction and reconstitution protocol is a powerful technique to study the role of each component of the thin filament. It is also useful for studying the properties of genetically modified molecules such as actin and tropomyosin. We also review the combination of this protocol with sinusoidal analysis, which will provide a solid technique for determining the effect of regulatory proteins on actomyosin interaction and concomitant cross-bridge kinetics. We suggest that thin filament-reconstituted muscle fibers are an ideal system for studying muscle physiology especially when gene modifications of actin or tropomyosin are involved.
\end{abstract}

\section{Introduction}

Striated muscle has a highly ordered structure where muscle proteins are arranged in an array of filaments $[1,2]$. It is critically important that these structures are maintained so that muscle proteins retain their physiological functions. Thus, physiological functions of muscle proteins must be studied using a model that preserves their native higherorder structure. Thin filaments involving actin, tropomyosin and troponin can be reconstituted in vitro which preserves $\mathrm{Ca}^{2+}$ sensitivity, and, in this model, the measurement of active tension or sliding speed is possible (in vitro motility assay system) $[3,4]$. In the in vitro motility assay system, the higher-order structure of thin filaments is reconstituted but myosin or the motor domain of myosin is randomly placed on glass-coverslip, which differs from the physiological condition. The higher-order structure of thick filaments can be reconstituted relatively easily in vitro by lowering the ionic strength of the solution [5] although force or sliding velocity could not be measured until single-molecule manipulation techniques were developed $[6,7]$. These in vitro experiments with reconstituted filaments revealed that muscle proteins show altered kinetics when the higherorder structure is conserved, indicating the importance of the higher-order structure in the study of muscle proteins. Although filament structures can be reconstituted in vitro using purified proteins, it is still difficult to reconstruct the 3D structure of muscle where thick and thin filaments are arranged in a hexagonal lattice. Recently, a new in vitro assay system was developed which used an isolated A-band and reconstituted actin filament to measure the active force developed in the thick filament lattice structure [8]. This approach enables the study of the physiology of muscle proteins preserving higher-order structure of muscle but, because the preparation of A-band is labor consuming and the setup is relatively complicated, this technique did not become widely used. Another approach is to exchange the small molecules such as troponin and myosin light chain in skinned muscle [9-12]. Although this approach enables the study of the properties of some muscle proteins, most other proteins such as myosin, actin, and tropomyosin are difficult to exchange; thus another approach is needed.

Removal and reconstitution of the filament in skinned muscle is another way to exchange muscle proteins with 


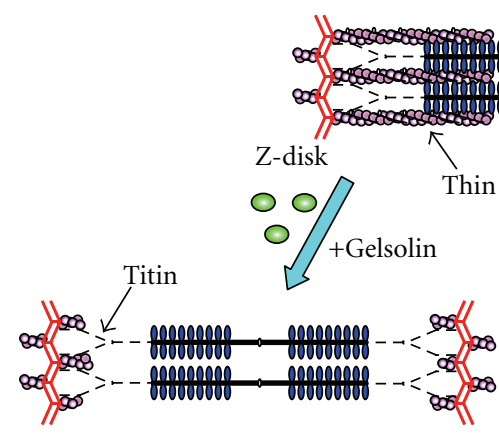

Thin filament-free muscle fiber

(b)

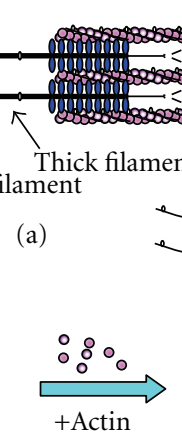

(a)

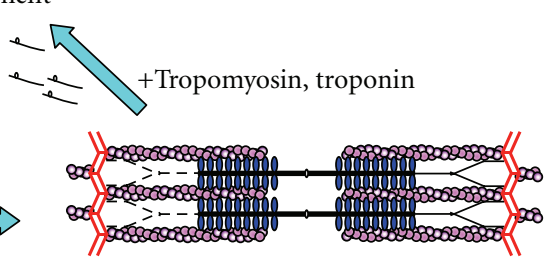

Actin-filament-reconstituted muscle fiber

(c)

FIGURE 1: Schematic diagram illustrating the thin filament removal and reconstitution protocol. By applying gelsolin to skinned muscle fibers (a), thin filament free muscle fibers (b) can be obtained. By adding actin monomer to these thin filament free muscle fibers, actin-filamentreconstituted muscle fibers without regulatory proteins (c) can be obtained. Thin filament can be reconstituted by adding regulatory proteins to these actin-filament-reconstituted muscle fibers.

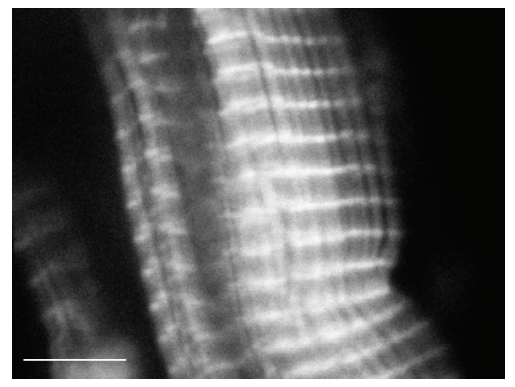

(a)

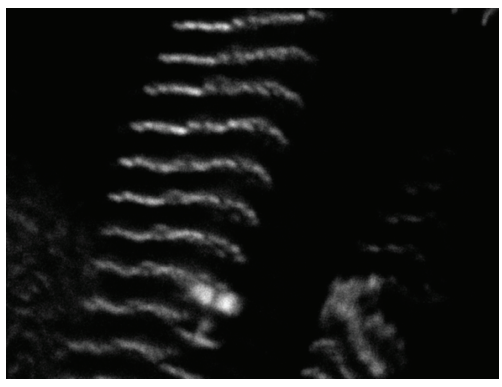

(b)

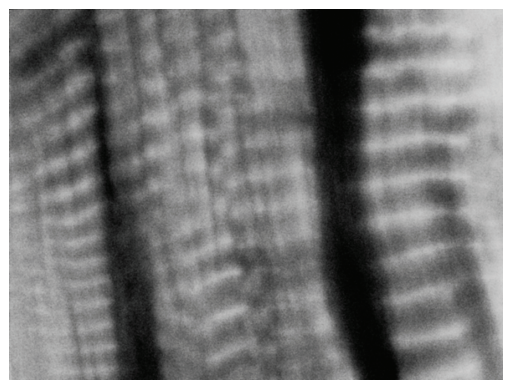

(c)

FIGURE 2: Confocal fluorescent micrograph of muscle fibers at each step of thin filament removal and reconstitution. (a) Control cardiac muscle fibers. (b) Gelsolin-treated muscle fibers. (c) Actin-filament-reconstituted muscle fibers. Actin filament was stained with rhodaminephalloidin. Scale bar: $5 \mu \mathrm{m}$.

purified proteins. Because thick filaments can be removed relatively easily by increasing the ionic strength [13], several attempts were made to reconstitute thick filaments using purified myosin. Although myosin filaments can be reconstituted relatively easily in vitro, reconstitution of thick filaments inside skinned muscle fibers is not possible due to the spontaneous polymerization of myosin filament. On the other hand, thin filaments cannot be removed by simply changing the ionic strength of the surrounding environment. Selective removal of thin filaments was achieved by using the actin filament severing protein, gelsolin, as a molecular tool, which enabled elimination of actin, troponin, and tropomyosin from skinned muscle fibers [14-16]. Because gelsolin requires $\mathrm{Ca}^{2+}$ for actin filament severing, actomyosin ATPase inhibitor was initially used during gelsolin treatment, which was followed by the use of actin filament severing domain of gelsolin [17]. Using these thin filament-removed (thin filament-free) skinned muscle fibers, reconstitution of thin filaments was performed [18]. Figure 1 illustrates the procedure for the removal and reconstitution of thin filaments. When purified actin monomer was added to thin filament-free skinned muscle fibers under the polymerizing condition, the actin filament polymerizes nucleated by a short fragment of the thin filament remaining at the Z-disk, reconstituting the actin filament. These investigators [18] used potassium iodide rather than potassium chloride and low temperature in order to slow the spontaneous nucleation of actin polymerization. These actin-filament-reconstituted muscle fibers can be used to study the properties of the actin filament without regulatory proteins. By the addition of the tropomyosin-troponin complex to actin-filamentreconstituted skinned muscle fibers, $\mathrm{Ca}^{2+}$ sensitivity was recovered [19]. Figure 2 shows confocal micrographs of muscle fibers stained for actin filament before and after the thin filament removal and after the reconstitution of actin filament. The confocal image clearly shows that thin filaments were removed by gelsolin treatment and restored after reconstitution of actin filament. When skinned skeletal muscle fibers were used for thin filament removal and reconstitution, only $\sim 20 \%$ of active tension was recovered regardless of the duration of actin filament reconstitution [18]. This was attributed to the damage of the Z-disk due to the overremoval of actin fragments which constitute the Z-disk and/or by proteases contaminated in the gelsolin solution. When skinned cardiac muscle was used, active tension was recovered by more than $100 \%$, average being 
$\sim 140 \%$ [19]. The successful recovery of active tension when cardiac muscle fibers were used was probably due to the stronger Z-disk structure of cardiac muscle. Active tension augmentation (by $\sim 40 \%$ ) was attributed to the length distribution of thin filaments in cardiac muscle, where length distribution is broad and the average length is shorter compared to skeletal muscle [20]. The longer length of thin filaments after reconstitution is likely to increase active force of the muscle fibers. When the skeletal tropomyosin-troponin complex was used in place of cardiac muscle, reconstituted muscle fibers showed a pCa-tension relationship that resembles that of the skeletal muscle fibers, showing successful exchange of the regulatory proteins.

The primary function of striated muscle is to generate active force; thus, when studying the physiological function of muscle proteins, it is important that active tension is measured. Unlike the in vitro motility assay system where expensive equipment, such as optical tweezers, is needed to measure force generation, it is comparatively easy to measure active force generated by thin filament-reconstituted skinned muscle fibers. Thus, thin filament-reconstituted muscle fibers are an ideal model for studying the physiology of thin filament proteins [21].

\section{Study of the Effect of Regulatory Proteins in Muscle Physiology Using Thin Filament- Reconstituted Skinned Muscle Fibers}

Unlike small proteins such as troponin or myosin light chains, tropomyosin is bound to seven actin monomers and it is extremely difficult to remove without damaging other muscle proteins. Using actin-filament-reconstituted muscle fibers before reconstitution of regulatory proteins, it is possible to measure the properties of bare actin filament without regulatory proteins in force generation. This is also possible by using in vitro motility experiments, but actin filaments are usually stained with fluorescent phalloidin, which may affect the active tension generating capability of actomyosin complex. Generally speaking, the in vitro motility system requires expensive experimental setups, such as optical tweezers, for force measurement, and the experiment is labor intensive, requiring numerous experiments to compensate for the large variation of the results. In addition, the in vitro motility assay system can only be performed at very low ionic strength, such as $\sim 50 \mathrm{mM}$, which is different from physiological conditions where ionic strength is $\sim 200 \mathrm{mM}$ [22]. In the actin-filamentreconstituted muscle model, active force generated by naked actin filament can be measured without phalloidin staining and at a physiological ionic strength. Moreover, the lattice structure of thick filament is conserved, offering a more natural physiological condition. Here, we show examples of the use of a thin filament removal and reconstitution protocol for studying the role of regulatory proteins in muscle physiology.

During muscle fatigue or the onset on ischemia, intracellular $\mathrm{pH}$ of muscle decreases, resulting in a decrease in active tension, $\mathrm{Ca}^{2+}$ sensitivity, and shortening velocity $[23,24]$.
It is known that even in skinned muscle fibers where only thick and thin filament is involved, active tension generation capability is affected by $\mathrm{pH}$, but whether regulatory protein affects the $\mathrm{pH}$ dependence of active tension is unknown. Using a thin filament removal and reconstitution protocol, the effect of regulatory proteins on the $\mathrm{pH}$ effect was studied [25]. In skinned cardiac muscle fibers, active tension increased linearly with an increase in $\mathrm{pH}$, consistent with previous reports $[26,27]$. This was also the case in actinfilament-reconstituted fibers, but the slope was much steeper than that with regulatory proteins. When regulatory proteins were reconstituted, the muscle fibers regained the original $\mathrm{pH}$-force relationship [25]. The $\mathrm{pH}$-force relationship was also recovered by the reconstitution of tropomyosin only, showing the importance of tropomyosin in the modulation of the $\mathrm{pH}$-force relationship of muscle. When regulatory proteins from skeletal muscle were used, the $\mathrm{pH}$-force relationship resembled that of skeletal muscle, which is larger than cardiac muscle. This result indicates that regulatory proteins protect muscle fibers from active tension decrease due to acidosis, especially in cardiac muscle where active tension loss by acidosis may be critical.

Sliding velocity and the active tension generation capability of striated muscle is also influenced by temperature where both the sliding velocity and active tension are larger at higher temperatures below $40^{\circ} \mathrm{C}[28,29]$. An increase in the sliding velocity indicates an increase in actomyosin ATPase activity, whereas an increase in active force suggests an increase in the number of force-generating cross-bridges. This shows that ATPase activity in the cross-bridge cycle is not equally affected by temperature [29], hypothesizing that the force generated by each cross-bridge does not change. Although the effect of temperature in muscle physiology has been well studied, the influence of regulatory proteins in the effect of temperature remains unknown. Using the thin filament removal and reconstitution protocol, the effect of regulatory proteins in temperature sensitivity of active force was studied [30]. In actin-filament-reconstituted muscle fibers without regulatory proteins, the effect of temperature on active tension was much less than the native fibers. When regulatory proteins were reconstituted, muscle fibers regained the temperature-force relationship of native fibers. Stiffness of muscle during contraction, an indicator of the amount of strong binding cross-bridges, decreased when temperature was increased in muscle fibers without regulatory proteins. Detailed study has revealed that cross-bridge detachment is enhanced by increasing temperature when regulatory proteins are absent [30]. This study showed that regulatory proteins may influence the actomyosin ATPase by modifying the surface of thin filaments.

One of the interesting features of striated muscle is its autooscillatory mechanism. Skinned striated muscle selfoscillates at few Hertz without $\mathrm{Ca}^{2+}$ oscillation in the presence of both MgADP and inorganic phosphate (Pi) at relaxing conditions (SPOC) $[30,31]$. Under these conditions, both active tension and sarcomere length oscillate periodically. To investigate whether tropomyosin is involved in the SPOC, actin-filament-reconstituted muscle fibers have been used [32]. When active tension was decreased 


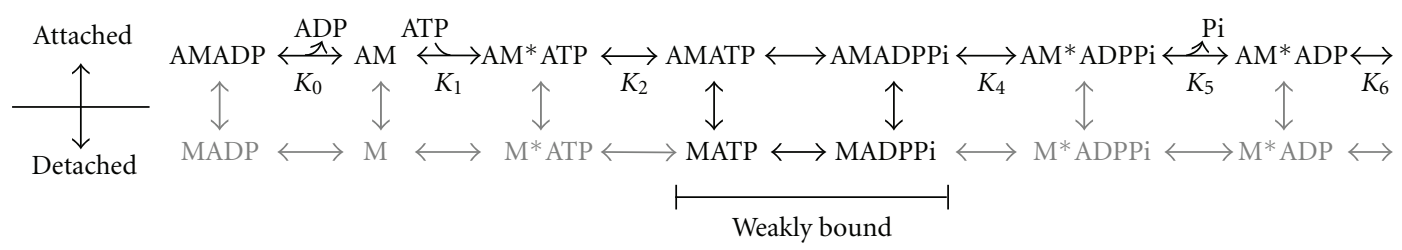

FIGURE 3: Kinetic model of actomyosin (AM) ATPase. The main pathway is shown in black and the minor pathway in grey. Upper row is in attached state and lower row is in detached state. A: actin; M: myosin. The asterisks show the difference in the conformation of the molecule.

by 2,3-butanedione 2-monoxime (BDM), the inhibitor of actomyosin ATPase, SPOC was observed in the presence of MgADP and Pi even when regulatory proteins were absent. This result clearly showed that tropomyosin is not required in SPOC and that oscillatory mechanisms are inherent in the actomyosin motor system. Recently, the mechanism of SPOC was clarified where the change in lattice spacing by mutual sliding of thick and thin filaments is the key point in the generation of oscillation [33]; this also indicated that the regulatory system is not a requirement for SPOC. Thus, the prediction made by the actin-filament-reconstituted model was shown to reflect the properties of muscle.

\section{Combination with Sinusoidal Analysis}

The thin filament removal and reconstitution protocol enables the exchange of actin and tropomyosin in skinned muscle fibers which is not possible in other methods, thus it is expected to be useful in studying the physiology of genetically engineered actin, troponin, and tropomyosin. Although the change in the active force by altering the surrounding environment such as $\mathrm{pH}$ or temperature is possible in the thin filament removal and reconstitution protocol, it is not possible to compare the active force before and after reconstitution of the actin filament because active tension recovery varies largely between experiments. This is problematic when the study of genetically engineered muscle protein is considered, because the first parameter that is to be compared is tension generation capability. To avoid this problem, it is ideal to measure the force per crossbridge. This can be achieved by measuring the stiffness of the muscle fibers during activation to determine the number of strongly bound cross-bridges and by measuring the kinetic parameters of the actomyosin ATPase scheme to calculate the number of cross-bridges among various states. To study the kinetics of actomyosin ATPase cycle, perturbation which can alter the ATPase kinetics at a speed faster than the reaction to be studied is needed. For example, temperature jump study (T-jump) can deduce several apparent rate constants relating to the actomyosin ATPase and by changing the concentration of MgATP, MgADP, or Pi, and, accordingly, the details of actomyosin ATPase can be presumed [28, 29]. The T-jump study revealed that muscle force generation is endothermic, and several cross-bridge states exist in actomyosin complex $[28,29]$. Other such "jump" experiments such as pressure [34], Pi [35, 36], or ATP [37, 38] can also deduce the elementary steps of the actomyosin ATPase. Perturbation to actomyosin ATPase can also be achieved by step length change; the kinetics were studied using both release and stretch protocols [39-41]. Other than applying step length change, sinusoidal length change was also used, which gives a higher signal-to-noise ratio than the step length change experiments $[42,43]$. Sinusoidal analysis revealed three exponential processes in response to length changes, which were attributed to six steps of the elementary steps of the cross-bridge cycle, and three equilibrium constants and four rate constants associated with this scheme were deduced [44]. Because sinusoidal analysis can characterize all of the equilibrium constants between the elementary steps of the cross-bridge cycle, calculation of the distribution of the cross-bridge species is possible. Measurement of the stiffness enables estimation of the amount of strong binding cross-bridges during activation, and consequently force per cross-bridge can also be estimated. Since sinusoidal analysis can reveal the details of the actomyosin ATPase cycle, the combination with the thin filament removal and reconstitution protocol is expected to yield many interesting results.

Investigations using the thin filament removal and reconstitution protocol and sinusoidal analysis revealed the influence of regulatory protein on the elementary steps of the cross-bridge cycle $[42,43]$. These studies showed that the regulatory proteins promote cross-bridge detachment by increasing $K_{2}$ and decreasing $K_{4}$, which was primarily caused by tropomyosin (Figure 3 ). This finding implies that the regulatory system inhibits actomyosin interaction even in the presence of $\mathrm{Ca}^{2+}$. At the same time, active force generated by each cross-bridge was larger in the presence of regulatory proteins, which may be induced by the conformational change of actin filaments due to the presence of regulatory proteins. The combination of the thin filament removal and reconstitution protocol and sinusoidal analysis is particularly useful in studying genetically modified actin and tropomyosin. Several actin mutants were studied in which the $\mathrm{N}$-terminal negative charges were changed using sinusoidal analysis [45]. Electrostatic force between the N-terminal charge of actin and the cationic loop 2 of myosin is believed to be important in the initial association with myosin. The result showed that the stronger negative charge at the $\mathrm{N}$-terminal enhances active force during contraction. The effect of genetically engineered tropomyosin on cross-bridge kinetics was also studied using the thin filament reconstitution protocol and sinusoidal analysis. Tropomyosin is a coiled-coil dimeric protein that binds to seven actin monomers and its sequence shows a 7 -fold repeat relating to the sites which bind to actin [46]. The seven 
repeats are quasi-equivalent but solution studies show the differences in their functions, where the first and last periods are important in the head-to-tail association of tropomyosin molecule and period 4 involved in troponin binding. Using the thin filament reconstitution protocol, Kawai et al. showed that period 3 of tropomyosin is essential in the enhancement of isometric tension [47]. The physiological properties of tropomyosin mutations (V95A, D175N, and E180G) regarding hypertrophic cardiomyopathy (HCM) were also studied using the same method and showed that contractility was decreased and relaxation was impaired in HCM tropomyosin [48]. Likewise, the effects of phosphorylation and/or dephosphorylation of tropomyosin were investigated by using this technique [49]. These studies showed that the combination of the thin filament reconstitution protocol and sinusoidal analysis is very useful in the study of genetically modified actin and tropomyosin.

\section{Studying Titin Using Thin Filament-Free Muscle Fibers}

Titin (also known as connectin) is a muscle-specific giant protein with a molecular weight of $3 \sim 4 \mathrm{MDa}$ [50]. Titin spans from Z-disk to M-line in half sarcomere constituting a third filament in muscle, and it functions as a molecular spring during stretch which determines the passive force. The property of titin is particularly important in cardiac muscle physiology because titin's property as a spring is a major determinant of diastolic force. Mechanical properties of titin can be studied by stretching muscle fibers during relaxation, but there is an effect from slow cycling weakly bound cross-bridges [51]. The effect of weakly bound crossbridges can be eliminated by using the thin filament-free muscle fibers, which is the state before the reconstitution of actin filament in the thin filament removal and reconstitution protocol. Figure 4 shows the stress-strain relationship of cardiac myofibril treated with gelsolin. When skinned skeletal muscle fibers were stretched after removal of thin filament, the stress-strain relationship differed from those in the presence of thin filament showing the presence of weaklybound cross-bridges [17]. The effect of $\mathrm{Ca}^{2+}$ on passive force was investigated using thin filament-free muscle fibers [52]; in the presence of $\mathrm{Ca}^{2+}$, it is important to eliminate the effect of cross-bridges to measure passive force, as the coexistence of $\mathrm{Ca}^{2+}$ and ATP will activate the cross-bridges. Studies have shown that $\mathrm{Ca}^{2+}$ increases the stiffness of titin, which may play a role during systole and diastole $[52,53]$.

\section{Conclusions}

The thin filament removal and reconstitution protocol is useful in the study of the physiology of actin and tropomyosin in muscle contraction. It is particularly valuable when combined with sinusoidal analysis. Actin-filament-reconstituted muscle fibers are useful in studying the properties of genetically modified actin. Thin filament-reconstituted model is helpful in studying the properties of tropomyo-sin. Muscle fibers without thin filament can be used to study the mechanical property of giant protein titin.

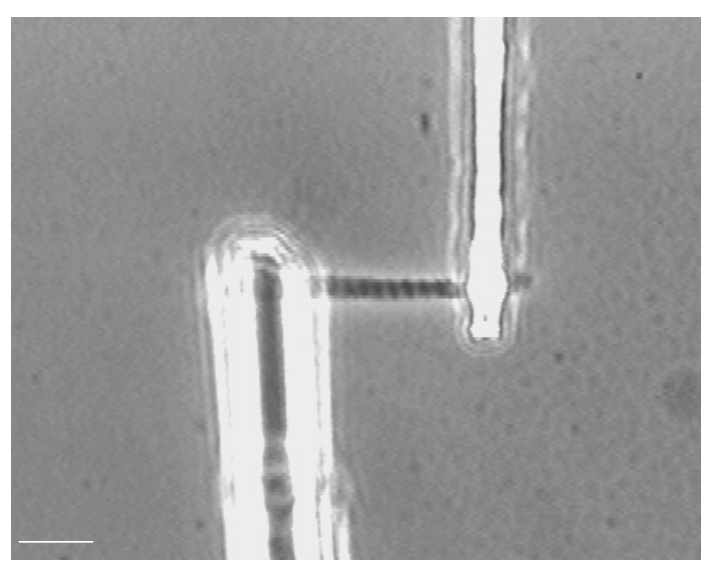

(a)

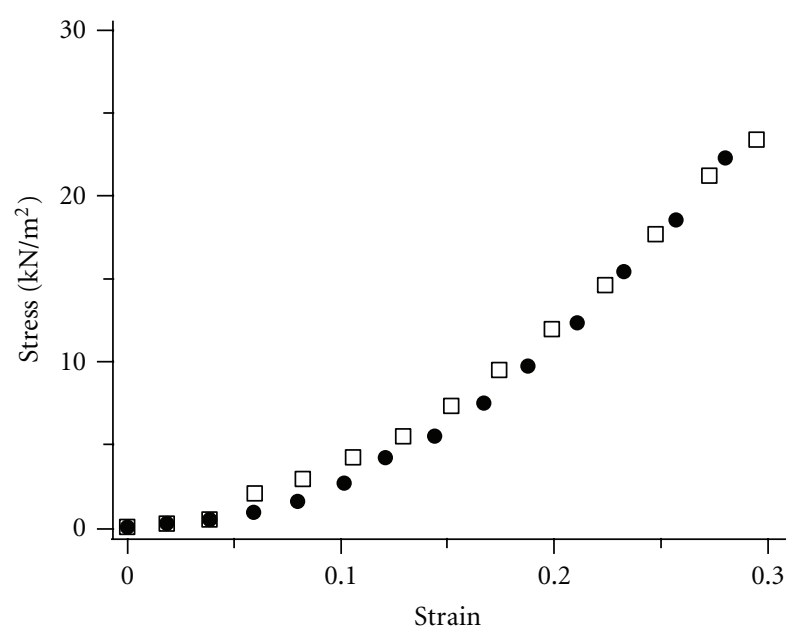

(b)

FIGURE 4: (a) Phase-contrast image of cardiac myofibril treated with gelsolin. Myofibril was held with two glass needles. Thick needle was moved to the left to stretch the myofibril and force was measured by the bending of the thin needle. Scale bar: $10 \mu \mathrm{m}$. (b) Stress-strain relationship of myofibril treated with gelsolin. Two examples are shown. Strain is expressed relative to the slack length.

\section{Acknowledgment}

The work of the authors was supported in part by Grantsin-Aid for Scientific Research (N.F. and S.K.), Scientific Research on Innovative Areas (N.F. and S.K.) and for Challenging Exploratory Research (N.F.) from the Ministry of Education, Culture, Sports, Science and Technology of Japan and by grants from the Japan Science and Technology Agency (CREST) (N.F.), the Vehicle Racing Commemorative Foundation (S.K.), the Institute of Seizon and Life Sciences (S.K.) and the CASIO Science Promotion Foundation (N.F.).

\section{References}

[1] A. F. Huxley and R. Niedergerke, "Structural changes in muscle during contraction: interference microscopy of living muscle fibres," Nature, vol. 173, no. 4412, pp. 971-973, 1954.

[2] H. Huxley and J. Hanson, "Changes in the cross-striations 
of muscle during contraction and stretch and their structural interpretation," Nature, vol. 173, no. 4412, pp. 973-976, 1954.

[3] A. M. Gordon, M. A. LaMadrid, Y. Chen, Z. Luo, and P. B. Chase, "Calcium regulation of skeletal muscle thin filament motility in vitro," Biophysical Journal, vol. 72, no. 3, pp. 1295 1307, 1997.

[4] E. Homsher, D. M. Lee, C. Morris, D. Pavlov, and L. S. Tobacman, "Regulation of force and unloaded sliding speed in single thin filaments: effects of regulatory proteins and calcium," Journal of Physiology, vol. 524, no. 1, pp. 233-243, 2000.

[5] R. Niederman and T. D. Pollard, "Human platelet myosin. II. In vitro assembly and structure of myosin filaments," Journal of Cell Biology, vol. 67, no. 1, pp. 72-92, 1975.

[6] A. Ishijima, Y. Harada, H. Kojima, T. Funatsu, H. Higuchi, and T. Yanagida, "Single-molecule analysis of the actomyosin motor using nano-manipulation," Biochemical and Biophysical Research Communications, vol. 199, no. 2, pp. 1057-1063, 1994.

[7] M. Kaya and H. Higuchi, "Nonlinear elasticity and an 8-nm working stroke of single myosin molecules in myofilaments," Science, vol. 329, no. 5992, pp. 686-689, 2010.

[8] M. Suzuki, H. Fujita, and S. Ishiwata, "A new muscle contractile system composed of a thick filament lattice and a single actin filament," Biophysical Journal, vol. 89, no. 1, pp. 321-328, 2005.

[9] M. Hatakenaka and I. Ohtsuki, "Effect of removal and reconstitution of troponins $\mathrm{C}$ and I on the ${ }^{2+}$-activated tension development of single glycerinated rabbit skeletal muscle fibers," European Journal of Biochemistry, vol. 205, no. 3, pp. 985-993, 1992.

[10] F. Shiraishi, M. Kambara, and I. Ohtsuki, "Replacement of troponin components in myofibrils," Journal of Biochemistry, vol. 111 , no. 1 , pp. $61-65,1992$.

[11] J. M. Metzger and R. L. Moss, "Myosin light chain 2 modulates calcium-sensitive cross-bridge transitions in vertebrate skeletal muscle," Biophysical Journal, vol. 63, no. 2, pp. 460-468, 1992.

[12] S. Lowey, G. S. Waller, and K. M. Trybus, "Skeletal muscle myosin light chains are essential for physiological speeds of shortening," Nature, vol. 365, no. 6445, pp. 454-456, 1993.

[13] H. Higuchi and S. Ishiwata, "Disassembly kinetics of thick filaments in rabbit skeletal muscle fibers. Effects of ionic strength, ${ }^{2+}$ concentration, $\mathrm{pH}$, temperature, and cross-bridges on the stability of thick filament structure," Biophysical Journal, vol. 47, no. 3, pp. 267-275, 1985.

[14] T. Funatsu, H. Higuchi, and S. Ishiwata, "Elastic filaments in skeletal muscle revealed by selective removal of thin filaments with plasma gelsolin," Journal of Cell Biology, vol. 110, no. 1, pp. 53-62, 1990.

[15] H. L. M. Granzier and K. Wang, "Interplay between passive tension and strong and weak binding cross-bridges in insect indirect flight muscle: a functional dissection by gelsolinmediated thin filament removal," Journal of General Physiology, vol. 101, no. 2, pp. 235-270, 1993.

[16] T. Funatsu, E. Kono, H. Higuchi et al., "Elastic filaments in situ in cardiac muscle: deep-etch replica analysis in combination with selective removal of actin and myosin filaments," Journal of Cell Biology, vol. 120, no. 3, pp. 711-724, 1993.

[17] H. L. M. Granzier and K. Wang, "Passive tension and stiffness of vertebrate skeletal and insect flight muscles: the contribution of weak cross-bridges and elastic filaments," Biophysical Journal, vol. 65, no. 5, pp. 2141-2159, 1993.
[18] T. Funatsu, T. Anazawa, and S. Ishiwata, "Structural and functional reconstruction of thin filaments in skeletal muscle," Journal of Muscle Research and Cell Motility, vol. 15, no. 2, pp. 158-171, 1994.

[19] H. Fujita, K. Yasuda, S. Niitsu, T. Funatsu, and S. Ishiwata, "Structural and functional reconstitution of thin filaments in the contractile apparatus of cardiac muscle," Biophysical Journal, vol. 71, no. 5, pp. 2307-2318, 1996.

[20] T. F. Robinson and S. Winegrad, "Variation of thin filament length in heart muscle," Nature, vol. 267, no. 5606, pp. 74-75, 1977.

[21] M. Kawai and S. Ishiwata, "Use of thin filament reconstituted muscle fibres to probe the mechanism of force generation," Journal of Muscle Research and Cell Motility, vol. 27, no. 5-7, pp. 455-468, 2006.

[22] D. W. Maughan and R. E. Godt, "A quantitative analysis of elastic, entropic, electrostatic, and osmotic forces within relaxed skinned muscle fibers," Biophysics of Structure and Mechanism, vol. 7, no. 1, pp. 17-40, 1980.

[23] M. J. Dawson, D. G. Gadian, and D. R. Wilkie, "Muscular fatigue investigated by phosphorus nuclear magnetic resonance," Nature, vol. 274, no. 5674, pp. 861-866, 1978.

[24] K. A. P. Edman and A. R. Mattiazzi, "Effects of fatigue and altered $\mathrm{pH}$ on isometric force and velocity of shortening at zero load in frog muscle fibres," Journal of Muscle Research and Cell Motility, vol. 2, no. 3, pp. 321-334, 1981.

[25] H. Fujita and S. Ishiwata, "Tropomyosin modulates $\mathrm{pH}$ dependence of isometric tension," Biophysical Journal, vol. 77, no. 3, pp. 1540-1546, 1999.

[26] S. P. Robertson and W. G. Kerrick, "The effects of $\mathrm{pH}$ on ${ }^{2+}$ activated force in frog skeletal muscle fibers," Pflugers Archiv European Journal of Physiology, vol. 380, no. 1, pp. 41-45, 1979.

[27] P. B. Chase and M. J. Kushmerick, "Effects of $\mathrm{pH}$ on contraction of rabbit fast and slow skeletal muscle fibers," Biophysical Journal, vol. 53, no. 6, pp. 935-946, 1988.

[28] K. W. Ranatunga, "Endothermic force generation in skinned cardiac muscle from rat," Journal of Muscle Research and Cell Motility, vol. 20, no. 5-6, pp. 489-496, 1999.

[29] Y. E. Goldman, J. A. McCray, and K. W. Ranatunga, "Transient tension changes initiated by laser temperature jumps in rabbit psoas muscle fibres," Journal of Physiology, vol. 392, pp. 71-95, 1987.

[30] H. Fujita and M. Kawai, "Temperature effect on isometric tension is mediated by regulatory proteins tropomyosin and troponin in bovine myocardium," Journal of Physiology, vol. 539, part 1, pp. 267-276, 2002.

[31] N. Fukuda, H. Fujita, T. Fujita, and S. Ishiwata, "Spontaneous tension oscillation in skinned bovine cardiac muscle," Pflugers Archiv European Journal of Physiology, vol. 433, no. 1-2, pp. $1-8,1996$.

[32] H. Fujita and S. Ishiwata, "Spontaneous oscillatory contraction without regulatory proteins in actin filament-reconstituted fibers," Biophysical Journal, vol. 75, no. 3, pp. 1439-1445, 1998.

[33] K. Sato, M. Ohtaki, Y. Shimamoto, and S. Ishiwata, "A theory on auto-oscillation and contraction in striated muscle," Progress in Biophysics and Molecular Biology, vol. 105, no. 3, pp. 199-207, 2011.

[34] N. S. Fortune, M. A. Geeves, and K. W. Ranatunga, "Contractile activation and force generation in skinned rabbit muscle fibres: effects of hydrostatic pressure," Journal of Physiology, vol. 474, no. 2, pp. 283-290, 1994. 
[35] J. A. Dantzig, Y. E. Goldman, N. C. Millar, J. Lacktis, and E. Homsher, "Reversal of the cross-bridge force-generating transition by photogeneration of phosphate in rabbit psoas muscle fibres," Journal of Physiology, vol. 451, pp. 247-278, 1992.

[36] E. Homsher and N. C. Millar, "Caged compounds and striated muscle contraction," Annual Review of Physiology, vol. 52, pp. 875-896, 1990.

[37] K. Horiuti, K. Kagawa, and K. Yamada, "Transient contraction of muscle fibers on photorelease of ATP at intermediate concentrations of ${ }^{2+}$," Biophysical Journal, vol. 67, no. 5, pp. 1925-1932, 1994.

[38] J. A. Dantzig, M. G. Hibberd, D. R. Trentham, and Y. E. Goldman, "Cross-bridge kinetics in the presence of MgADP investigated by photolysis of caged ATP in rabbit psoas muscle fibres," Journal of Physiology, vol. 432, pp. 639-680, 1991.

[39] G. Piazzesi, F. Francini, M. Linari, and V. Lombardi, "Tension transients during steady lengthening of tetanized muscle fibres of the frog," Journal of Physiology, vol. 445, pp. 659-711, 1992.

[40] G. Piazzesi, M. Linari, M. Reconditi, F. Vanzi, and V. Lombardi, "Cross-bridge detachment and attachment following a step stretch imposed on active single frog muscle fibres," Journal of Physiology, vol. 498, no. 2, part 1, pp. 3-15, 1997.

[41] M. Linari, V. Lombardi, and G. Piazzesi, "Cross-bridge kinetics studied with staircase shortening in single fibres from frog skeletal muscle," Journal of Muscle Research and Cell Motility, vol. 18, no. 1, pp. 91-101, 1997.

[42] H. Fujita, D. Sasaki, S. Ishiwata, and M. Kawai, "Elementary steps of the cross-bridge cycle in bovine myocardium with and without regulatory proteins," Biophysical Journal, vol. 82, no. 2, pp. 915-928, 2002.

[43] H. Fujita, X. Lu, M. Suzuki, S. Ishiwata, and M. Kawai, "The effect of tropomyosin on force and elementary steps of the cross-bridge cycle in reconstituted bovine myocardium," Journal of Physiology, vol. 556, part 2, pp. 637-649, 2004.

[44] M. Kawai and H. R. Halvorson, "Two step mechanism of phosphate release and the mechanism of force generation in chemically skinned fibers of rabbit psoas muscle," Biophysical Journal, vol. 59, no. 2 I, pp. 329-342, 1991.

[45] X. Lu, M. K. Bryant, K. E. Bryan, P. A. Rubenstein, and M. Kawai, "Role of the N-terminal negative charges of actin in force generation and cross-bridge kinetics in reconstituted bovine cardiac muscle fibres," Journal of Physiology, vol. 564, part 1, pp. 65-82, 2005.

[46] A. D. McLachlan and M. Stewart, "The 14 fold periodicity in $\alpha$ tropomyosin and the interaction with actin," Journal of Molecular Biology, vol. 103, no. 2, pp. 271-293, 1976.

[47] M. Kawai, X. Lu, S. E. Hitchcock-Degregori et al., "Tropomyosin period 3 is essential for enhancement of isometric tension in thin filament-reconstituted bovine myocardium," Journal of Biophysics, vol. 2009, Article ID 380967, 17 pages, 2009.

[48] F. Bai, A. Weis, A. K. Takeda et al., "Enhanced active cross-bridges during diastole: molecular pathogenesis of tropomyosin's HCM mutations," Biophysical Journal, vol. 10, no. 4 , pp. 1013-1023, 2011.

[49] X. Lu, D. H. Heeley, L. B. Smillie, and M. Kawai, "The role of tropomyosin isoforms and phosphorylation in force generation in thin-filament reconstituted bovine cardiac muscle fibres," Journal of Muscle Research and Cell Motility, vol. 31, no. 2, pp. 93-109, 2010.

[50] S. Labeit and B. Kolmerer, "Titins: giant proteins in charge of muscle ultrastructure and elasticity," Science, vol. 270, no. 5234, pp. 293-296, 1995.
[51] R. Horowits, E. S. Kempner, M. E. Bisher, and R. J. Podolsky, "A physiological role for titin and nebulin in skeletal muscle," Nature, vol. 323, no. 6084, pp. 160-164, 1986.

[52] H. Fujita, D. Labeit, B. Gerull, S. Labeit, and H. L. Granzier, "Titin isoform-dependent effect of calcium on passive myocardial tension," American Journal of Physiology, vol. 287, no. 6, pp. H2528-H2534, 2004.

[53] D. Labeit, K. Watanabe, C. Witt et al., "Calcium-dependent molecular spring elements in the giant protein titin," Proceedings of the National Academy of Sciences of the United States of America, vol. 100, no. 23, pp. 13716-13721, 2003. 

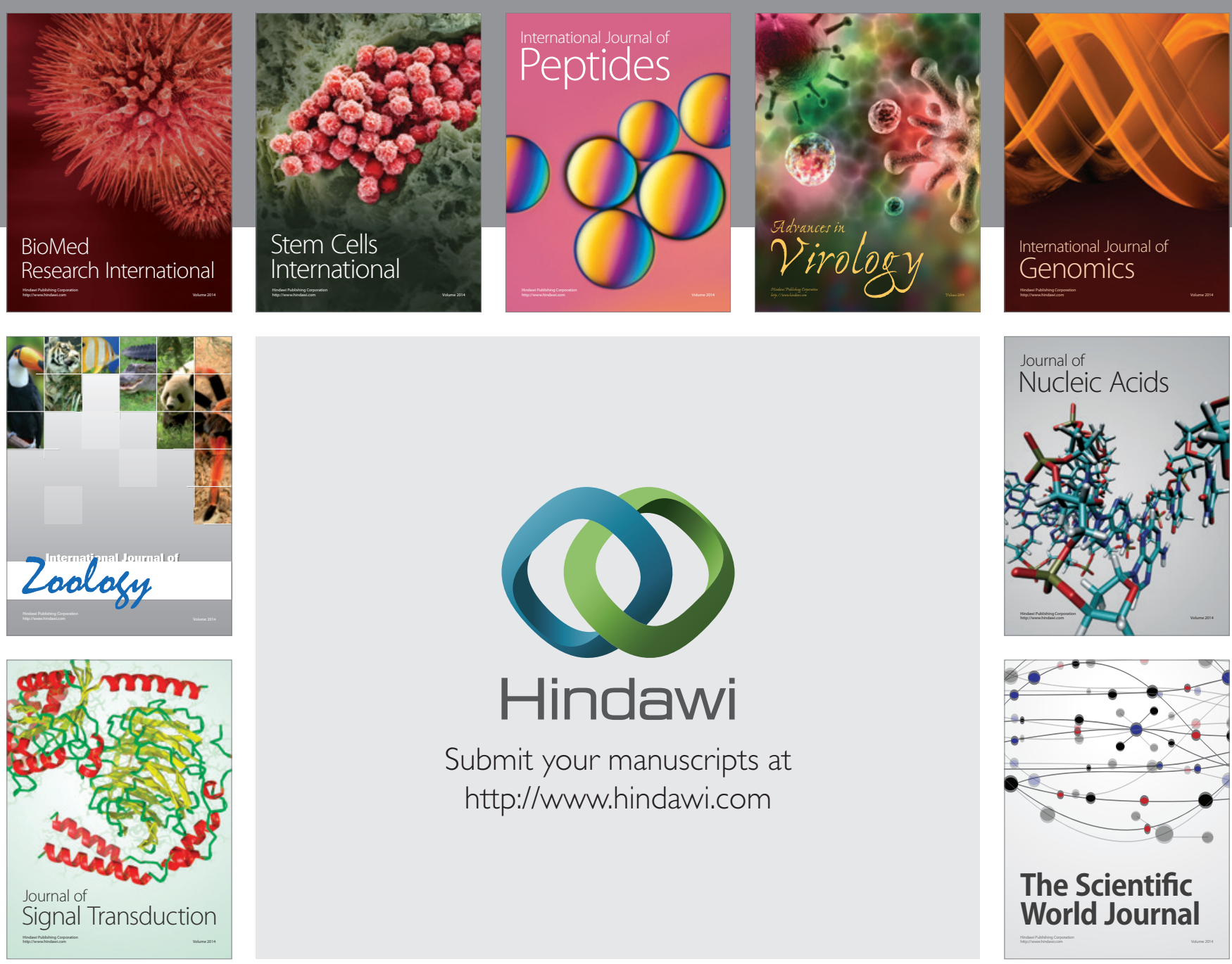

Submit your manuscripts at

http://www.hindawi.com
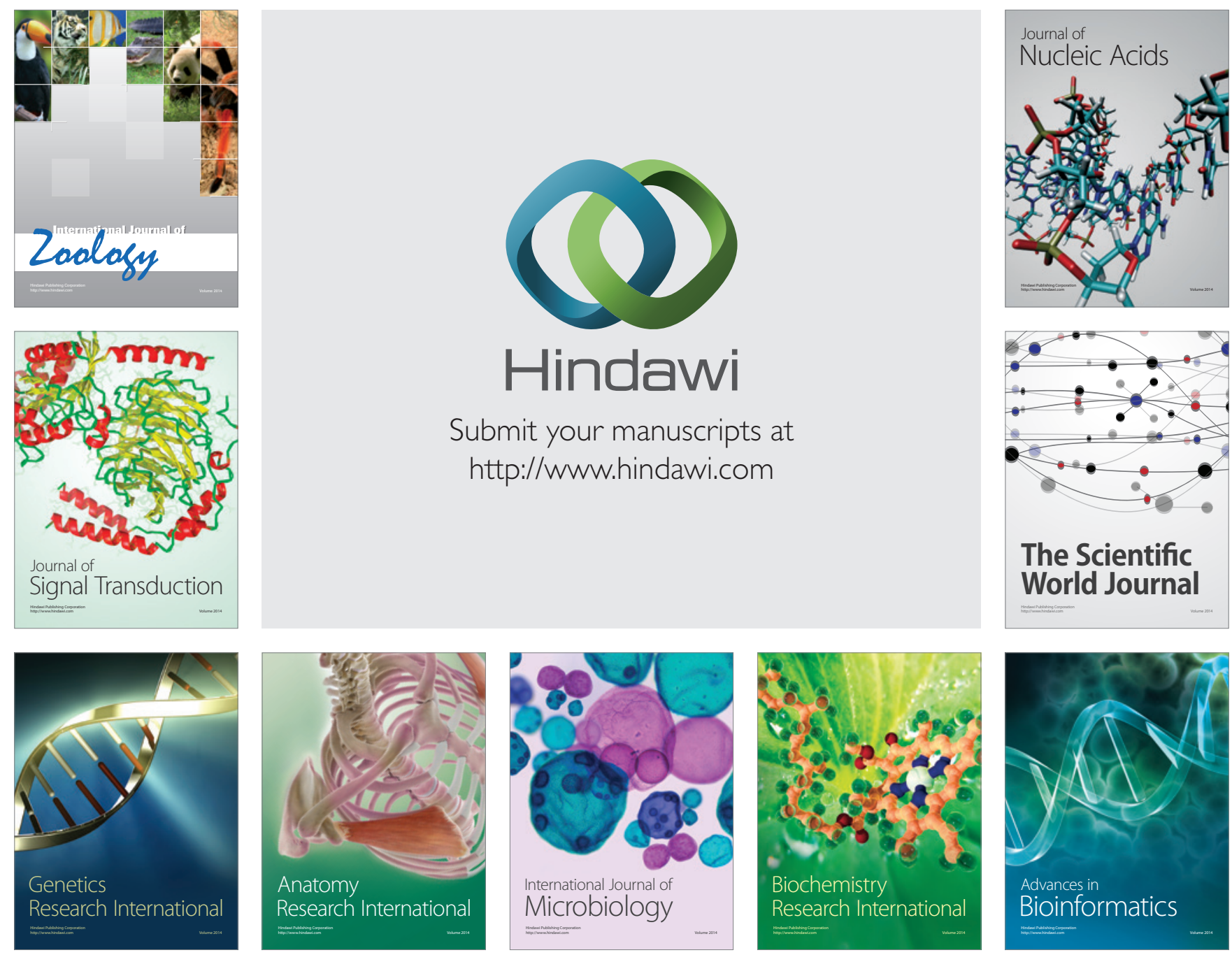

The Scientific World Journal
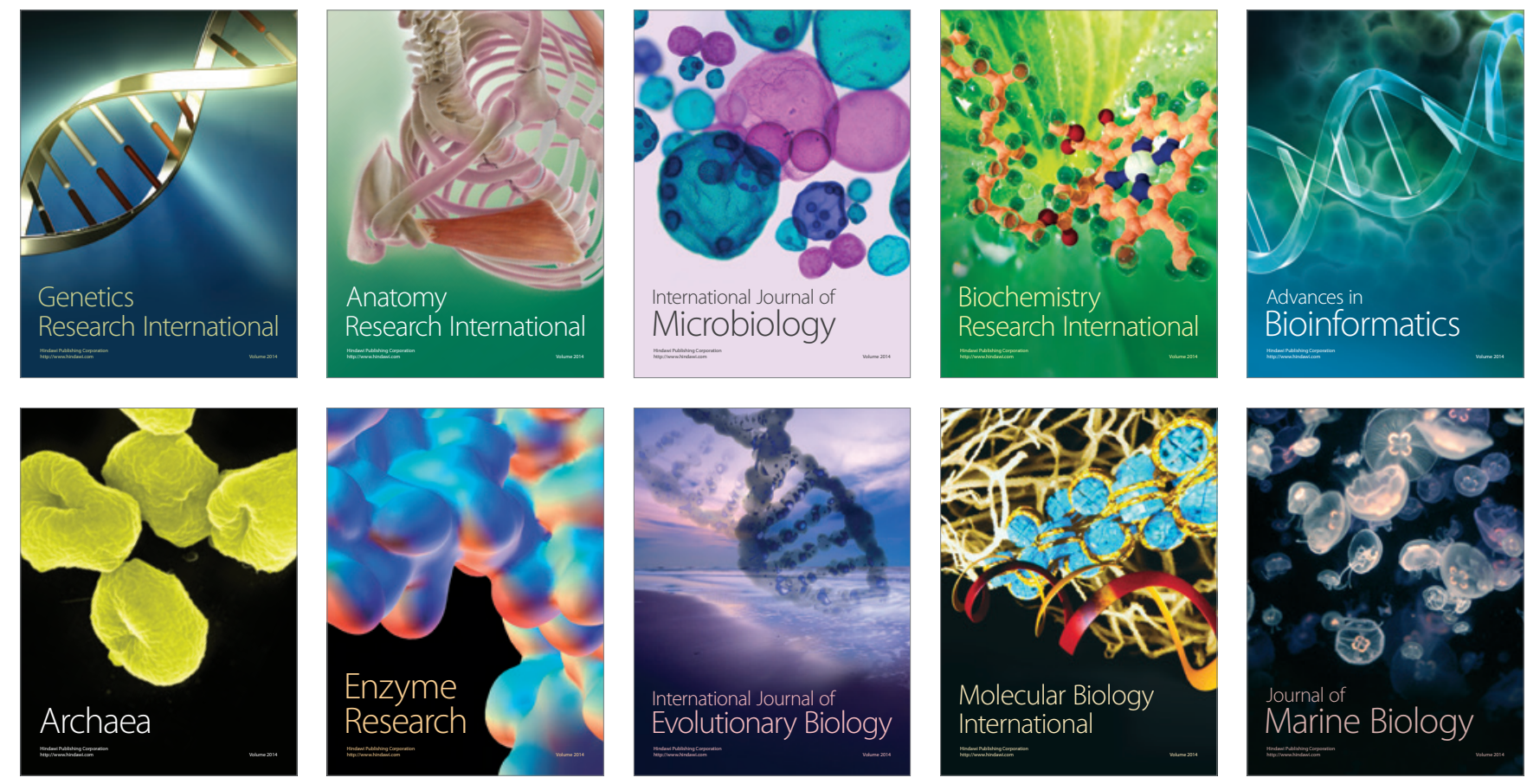